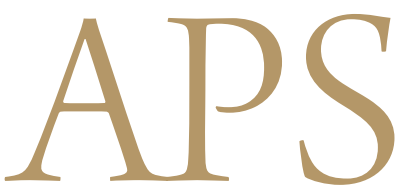

Archives of Plastic Surgery

\title{
Application of a Silicone Sheet in Negative- Pressure Wound Therapy to Treat an Abdominal Wall Defect after Necrotizing Fasciitis
}

\author{
Jin Su Shin ${ }^{1}$, Hwan Jun $\mathrm{Choi}^{2}$ \\ ${ }^{1}$ Department of Plastic and Reconstructive Surgery, Soonchunhyang University College of Medicine, Bucheon; ${ }^{2}$ Department of Plastic and \\ Reconstructive Surgery, Soonchunhyang University College of Medicine, Cheonan, Korea
}

Necrotizing fasciitis (NF) is an aggressive soft-tissue infection involving the deep fascia and is characterized by extensive deterioration of the surrounding tissue. Immediate diagnosis and intensive treatment, including debridement and systemic antibiotics, represent the most important factors influencing the survival of NF patients. In this report, we present a case of $\mathrm{NF}$ in the abdomen due to an infection caused by a perforated small bowel after abdominal liposuction. It was successfully treated using negative-pressure wound therapy, in which a silicone sheet functioned as a barrier between the sponge and internal organs to protect the small bowel.

Keywords Fasciitis, necrotizing / Negative-pressure wound therapy / Peritoneum
Correspondence: Hwan Jun Choi Departement of Plastic and Reconstructive Surgery, Soonchunhyang University Cheonan Hostpital, 31 Sooncheonhyang 6-gil, Dongnam-gu, Cheonan 31151, Korea Tel: +82-41-570-2195

Fax: +82-41-574-6133

E-mail: medi619@hanmail.net

No potential conflict of interest relevant to this article was reported.

Received: 31 Jul $2016 \bullet$ Revised: 13 Oct $2016 \bullet$ Accepted: 18 Oct 2016

pISSN: 2234-6163・elSSN: 2234-6171 • https://doi.org/10.5999/aps.2017.44.1.76• Arch Plast Surg 2017;44:76-79

\section{INTRODUCTION}

Necrotizing fasciitis (NF) is a rapid aggressive infection of the soft tissue characterized by widespread necrosis of the fascia and subcutaneous tissue with initial sparing of the skin and muscle. Early diagnosis with prompt surgical debridement is essential for managing this life-threatening disease [1]. Negative-pressure wound therapy (NPWT) is a well-known wound care system for the management of complex wounds. Herein, we report a case of NF in the abdomen due to an infection caused by a perforated small bowel after abdominal liposuction that was successfully treated using NPWT and a silicone sheet. The silicone sheet was applied as a temporary barrier to minimize the damage to the small bowel by direct contact with the sponge.

\section{CASE}

A 56-year-old woman with no underlying disease presented to the emergency department with a 2-day history of severe abdominal pain and fever. Three days previously she had undergone abdominal liposuction at a local clinic. At presentation, the patient had a fever, with a temperature of $38.3^{\circ} \mathrm{C}$, and was hemodynamically unstable with a blood pressure of $90 / 50 \mathrm{~mm}$ $\mathrm{Hg}$ and a heart rate of $76 \mathrm{bpm}$. On physical examination, skin discoloration around the central portion of the abdomen with tense edema (Fig. 1A) and hyperactive bowel sounds were present. Rebound and rigidity were positive in all quadrants of the abdomen. The initial laboratory evaluation revealed leukocytosis $\left(13.4 \times 10^{3} / \mu \mathrm{L}\right)$, an elevated C-reactive protein level (51.1 


\section{Fig. 1. Necrotizing fasciitis of the abdomen}

(A) A 56-year-old who had undergone abdominal liposuction at a local clinic presented to the emergency department with severe pain, discoloration, and tense edema around the abdomen. $(B, C)$ Surgical findings revealed extensive necrosis of the subcutaneous fat and fascia of the abdominal wall and leakage of bilious fluid from a defect in the small bowel.
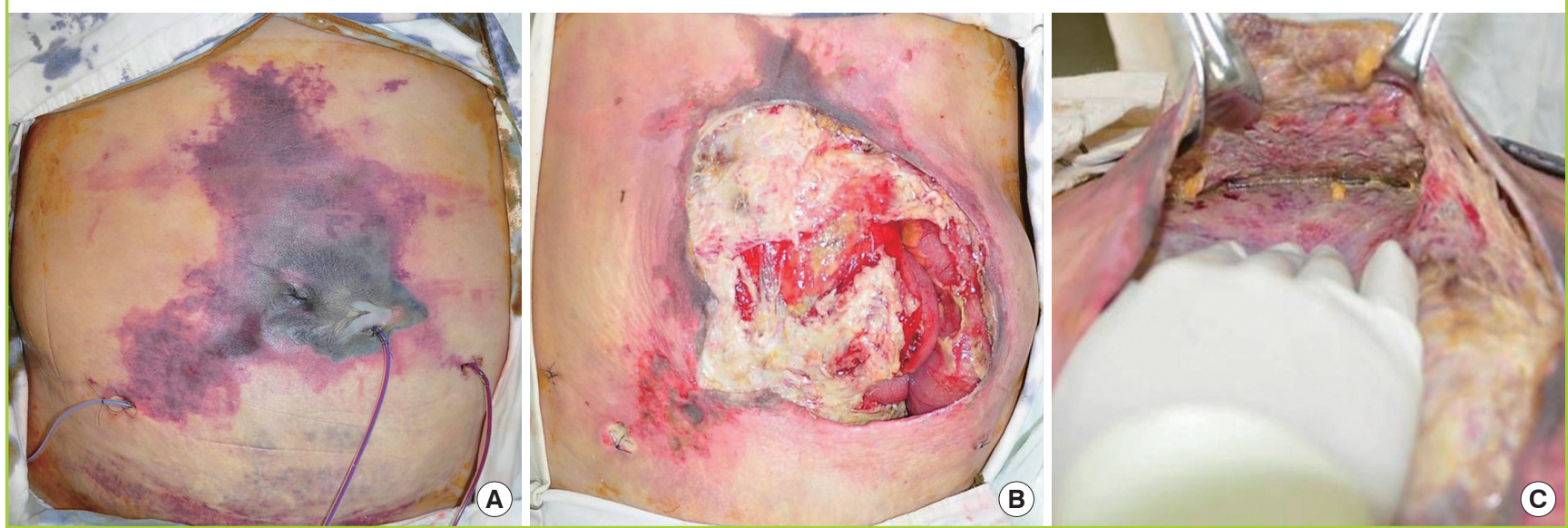

\section{Fig. 2. NPWT with a reinforced silicone sheet}

$(A, B)$ A reinforced silicone sheet and NPWT were applied to the wound for protection of the small bowel. Mepitel covered the rest of the raw surface where the small bowel was not exposed. NPWT, negative-pressure wound therapy.
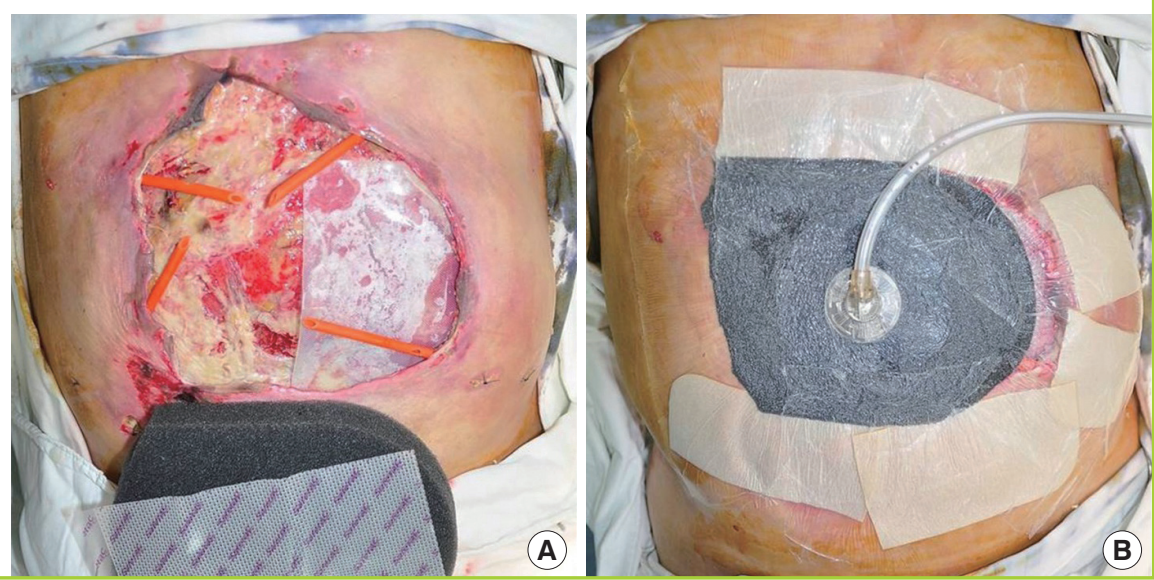

$\mathrm{mg} / \mathrm{L})$, and an elevated erythrocyte sedimentation rate (10 $\mathrm{mg} / \mathrm{L})$, with an abnormally low hemoglobin level $(8.1 \mathrm{~g} / \mathrm{dL})$. Her serum creatine kinase level was elevated $(3,022 \mathrm{U} / \mathrm{L})$. Initial computed tomography findings showed fluid collection and a free air pocket in the subcutaneous fat layer of the abdominal wall.

Under strong suspicion of NF resulting from liposuction, an emergency operation (excision of necrotic skin and debridement) was performed. The surgical findings were highly suggestive of NF, and included liquefied necrosis of fat layers and loss of normal resistance of the fascia to finger dissection. Furthermore, the small bowel was directly exposed with intestinal perforation. Extensive necrosis of the abdominal wall fascia with leakage of bilious fluid from a defect in the small bowel was observed during the operative procedure for abdominal wall debridement (Fig. 1B, C). General surgeons performed primary closure of the perforation but fluid leakage was detected again 2 days after the initial operation because the perforation was not completely sealed due to inflammation around the wound. The patient was intensively treated in the acute aggravation period by careful monitoring and coverage with broad-spectrum antibiotics. When the severe infection subsided with daily wound irrigation, 6 days after admission, the authors planned to cover the wound defect with NPWT (CuraVAC, CGBio, Hwaseong, Korea) to remove exudates and to promote wound healing. However, due to the full-thickness defect of the abdominal wall and peritoneum, directly applying the sponge to the small bowel was inevitable, which could induce a bowel injury. As a replacement for the peritoneum, a 0.03-inch thick reinforced silicone sheet (Bioplexus, Ventura, CA, USA) was applied beneath the sponge to minimize the damage to the small bowel and to set up an effective environment for exudates removal (Fig. 2). Under local anesthesia, serial surgical debridement and change of the NPWT dressing were performed 3 times a week for 4 weeks. 


\section{Fig. 3. Wound covered by a mesh STSG}

The wound was markedly improved with visible granulation tissue (A) and finally covered with a mesh STSG (B). (C) Most of the wound was closed, except a small defect. STSG, split-thickness skin graft.

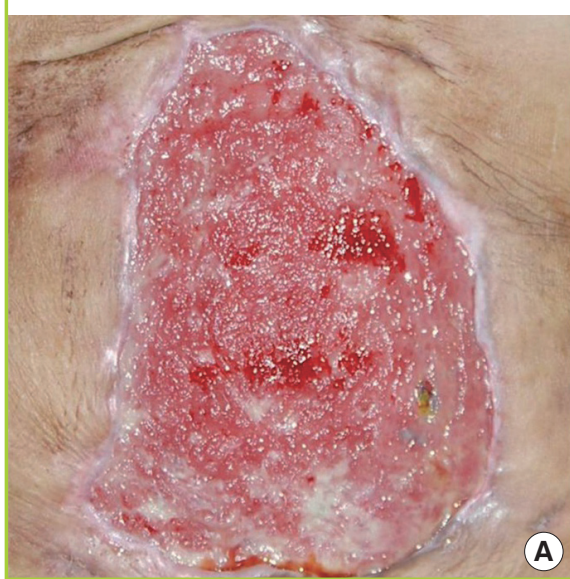

A

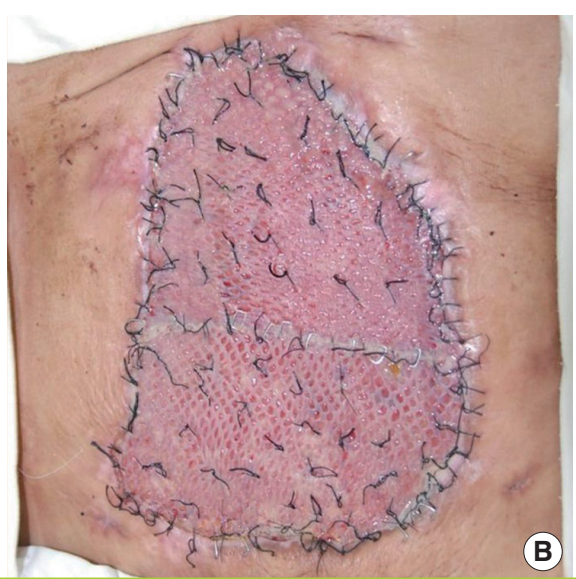

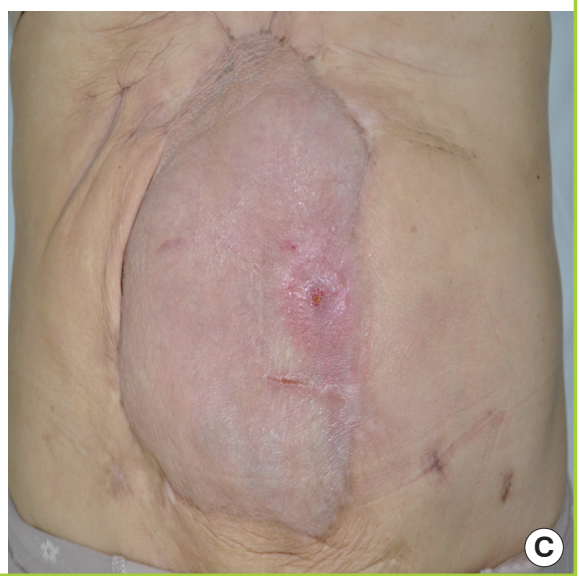

The silicone sheet was trimmed to match the size of the defect, as it diminished in size over time. Following the application of NPWT, set at $100 \mathrm{~mm} \mathrm{Hg}$ of continuous negative pressure, extensive debridement and NPWT successfully contributed to wound bed cleansing. Sequential wound assessment demonstrated marked improvement and reduction in wound size with visible granulation tissue that had started to contract, covering the defect. The open wound shrunk in size significantly with no complications such as an enterocutaneous fistula. Finally, after 4 weeks of NPWT, the wound beds were deemed suitable for grafting and finally covered with a mesh split-thickness skin graft (Fig. 3A, B). Fifty days after admission, the infection was cured, and most of the previous wound was closed except for a small defect that could be healed by simple dressing (Fig. 3C). The patient was discharged and received regular followed-up at the outpatient department for 12 months without complications.

\section{DISCUSSION}

NF is a rare, extremely aggressive condition that can lead to extensive soft tissue necrosis and sepsis. It spreads along the fascial planes, involving the subcutaneous tissue and skin. Although great improvements in treatment have been made, the mortality rate is still high $(25 \%-73 \%)$, meaning that prompt recognition, administration of broad-spectrum systemic antibiotics, and extensive surgical removal of devitalized tissues are necessary for successful treatment $[1,2]$. Most patients initially experience severe pain and tenderness with fever. The disease can be confirmed by intraoperative findings such as grayish necrotic deep fascia, lack of bleeding of the fascia, a lack of resistance to blunt dissection, and the presence of a foul odor with pus [3].
NPWT has a complementary function with a wide range of indications in the treatment of complex wounds. The application of NPWT can accelerate wound healing, because optimized blood flow increases tissue perfusion, enhances granulation formation, reduces bacterial contamination, diminishes toxin absorbance, promotes removal of excessive fluid from the wound bed, securely preserves residual vital tissues in the wound, and stimulates angiogenesis [4]. Furthermore, negative pressure helps eliminate fistulous tracts that result from necrotic fascia removal. Accumulating evidence suggests that NPWT is useful for the management of infected wounds, including NF [5]. Recently, an open abdomen NPWT system (ABThera, KCI Europe, Amstelveen, Netherlands) demonstrated valuable results, with faster achievement of primary closures than was obtained using abdominal dressing [6].

In this case, the patient was treated successfully with NPWT implemented following current standards. In particular, a 0.03-inch-thick reinforced silicone sheet was applied to function as a barrier and as a substitute for the peritoneum, preventing the exposed small bowel from coming into direct contact with the sponge and minimizing damage to the bowel. The silicone sheet was made of a flexible, translucent, vulcanized, silicone elastomer material with high degree of biocompatibility that is easy to apply and remove with minimal trauma. After resolution of the wound infection and the emergence of visible healthy granulation tissue, which took 4 weeks in this case, the NPWT and the silicone sheet can be removed to proceed to the next step for the complete coverage of the defect. This may be another good treatment option for the management of infected wounds using NPWT when the wound needs to be separated for protection and the effective removal of exudates.

In the light of the findings presented above, we emphasize the 
advantages of NPWT and the use of a silicone sheet, which successfully expedited wound healing in our patient with NF. Furthermore, careful postoperative follow-up assessment for the early diagnosis of complications such as intestinal perforation and NF is of paramount importance. The operating surgeon should be cognizant of the potential life-threatening complications of this condition.

\section{REFERENCES}

1. Ryssel H, Germann G, Kloeters O, et al. Necrotizing fasciitis of the extremities: 34 cases at a single centre over the past 5 years. Arch Orthop Trauma Surg 2010;130:1515-22.

2. Taviloglu K, Yanar H. Necrotizing fasciitis: strategies for di- agnosis and management. World J Emerg Surg 2007;2:19.

3. Naqvi GA, Malik SA, Jan W. Necrotizing fasciitis of the lower extremity: a case report and current concept of diagnosis and management. Scand J Trauma Resusc Emerg Med 2009; 17:28.

4. Huang C, Leavitt T, Bayer LR, et al. Effect of negative pressure wound therapy on wound healing. Curr Probl Surg 2014;51: 301-31.

5. Phelps JR, Fagan R, Pirela-Cruz MA. A case study of negative pressure wound therapy to manage acute necrotizing fasciitis. Ostomy Wound Manage 2006;52:54-9.

6. Olona C, Caro A, Duque E, et al. Comparative study of open abdomen treatment: ABThera vs. abdominal dressing. Hernia 2015;19:323-8. 